\title{
La tabla periódica en el Journal of Chemical Education a través del siglo $\mathrm{XX}$
}

\author{
Rita Linares* \\ Mercè Izquierdo**
}

Artículo recibido: 22-2-2007 y aprobado: 2-5-2007

The periodic table in the Journal of Chemical Education along (Through) the 20th Century

Resumen: Ante la amplia literatura con la que se encuentra quien comienza una investigación, presentamos esta revisión cronológica de 109 artículos relacionados con la tabla periódica (TP), publicados en el Journal of Chemical Education (JCE) a lo largo del siglo $\mathrm{XX}$, que puede servir de guía hacia otras fuentes a quienes se inician en este apasionante tema de los elementos químicos y su organización en la TP, según sea el interés particular de cada quien. Esta revisión incluye también la del concepto elemento, el cual, en los albores del siglo XXI, sigue teniendo más de una definición. Sin embargo, este trabajo no constituye un análisis histórico social del desarrollo de los conceptos aquí tratados.

Palabras clave: tabla periódica, elemento químico, Journal of Chemical Education.
Abstract: This paper presents a review of 109 articles about the periodic table, published in the Journal of Chemical Education (JCE) since 1924 until 2003, may save time to those who begin their search around the chemical elements and their organization in the periodic table. This review also includes the different definitions of the concept "chemical element".

Key words: periodic table, chemical element, Journal of Chemical Education.

\footnotetext{
Doctora en Didáctica de las Ciencias Experimentales, Universidad Autónoma de Barcelona. Profesora del Instituto de Educación y Pedagogía, Universidad del Valle.

** Doctora en Química y profesora de la Universidad Autónoma de Barcelona.
} 


\section{Introducción}

La TP de Mendeleiev incentivó tanto el trabajo de los químicos como el de los físicos que buscaban cada vez con más ahínco esclarecer la estructura interna de los átomos (Sandford, 1911; Bensaude-Vincent y Stengers, 1997; Scerri, 1998) y encontrar la base intrínseca de la periodicidad. En 1904, J. J. Thomson proponía la primera explicación física de la periodicidad, sugiriendo que los elementos con una configuración electrónica similar tendrían propiedades similares (Scerri, 1998). Y en 1913, como dicen Bensaude-Vincent y Stengers (1997, p. 196): "Bohr encaja todas las piezas del rompecabezas. El carácter periódico de la tabla de Mendeleiev puede explicarse por el limitado número de electrones que ocupan una misma órbita: cuando una órbita está llena, se cambia de línea en la tabla".

A partir de los estudios de rayos $X$ realizados por Henry Moseley en 1912, y que dieron origen a la ley que lleva su nombre, un elemento quedó definido por su número atómico (Z). En los siguientes veinte años se dispararon los descubrimientos y teorías que fueron conformando la teoría atómica que hoy conocemos y la base de la periodicidad encontrada por Mendeleiev salió a la luz. Según Kolodkine (1963), "desde 1913, seis años después de la muerte de Mendeleiev, la ley periódica ha sido explicada y confirmada de una manera ejemplar por la propia estructura del átomo". A partir de entonces, y especialmente durante la primera mitad del siglo $\mathrm{XX}$, muchos de los artículos referentes a la TP lo destacan una y otra vez (Courtines, 1925; Monroe y Turner, 1926; Ebel, 1938; Luder, 1939; Luder, 1943;
Babor, 1944; Emerson, 1944; Wagner y Simmons Booth, 1945; Szabo y Lakatos, 1957; Fernelius, 1986). En 1928 Conant (p. 25) escribía:

Las nuevas física y química de muchas maneras están centradas alrededor de la TP. En vez de asignar a cada elemento una posición en virtud de su peso atómico, ahora utilizamos los resultados de los rayos X para ordenarlos de acuerdo con su número atómico. [...] Las series de acuerdo al número atómico coinciden exactamente con las anteriores, excepto porque el telurio y el yodo, el argón y el potasio, y el cobalto y el níquel ya no están invertidos, sino que quedan en el lugar correcto. Las propiedades químicas de los elementos son una función periódica de sus posiciones en una serie determinada por su espectro de rayos $\mathrm{X}$. A diferencia de la ley periódica anterior basada en los pesos atómicos, esta es exacta. El número del correspondiente a la posición del elemento en las nuevas series es llamado número atómico. La nueva ley periódica debe formularse entonces como: las propiedades químicas de los elementos son una función periódica de sus números atómicos.

En su serie de tres artículos sobre el sistema periódico y la estructura atómica, Wiswesser (1945) ratifica lo manifestado anteriormente y afirma que la mecánica de ondas es una herramienta básica para la comprensión de la materia. Asevera que provee la única explicación lógica para: a) la interacción materia-energía, b) estructura atómica, c) el sistema periódico, d) estructura de valencia y estructura molecular, e) la causa de la acción química, la estabilidad o la inestabilidad y f) la naturaleza del cambio químico, defendiendo así la presentación 
del sistema periódico a través de la mecánica cuántica y no por el "histórico o empírico camino de los pesos atómicos”. Wiswesser explica el llenado de orbitales haciendo uso del orden creciente de $(n+l$ ) lo cual justifica el llenado del orbital $4 \mathrm{~s}$ $(4,0)$ antes del 3d $(3,2)$, y agrega: "Quienes reconocen el profundo significado del sistema periódico pueden impresionarse por este método puramente físico y matemático de determinar su forma a partir de principios realmente básicos, que no requiere la memorización de un solo hecho químico".

Sin embargo, puede decirse que a finales del siglo XX parece haber una tendencia a retomar la importancia de la TP como reflejo de una ley del comportamiento químico y no como una repetición ordenada de números (Izquierdo, 2002; Ludwig, 1992). Tanto Ludwig como Strong (1986) y Mabrouk (2003) aseguran que el mejor método mnemotécnico para aprender el principio de aufbau y recordar las configuraciones electrónicas es la TP misma, y concluye diciendo: "Usar la TP exclusivamente para racionalizar la distribución electrónica tiene la ventaja de enfatizar en los aspectos experimentales de la ciencia y evitar dar la impresión de que la química es nada más que un conjunto de reglas misteriosas".

En cuanto a la búsqueda de un sistema ordenador mejor que el de Mendeleiev, en la primera edición en francés de su libro Principios, él ya comenta sobre otros siete sistemas propuestos recientemente. Desde entonces los químicos no han dejado de proponer nuevos formatos, aunque sin encontrar ninguno que mejore el basado en el de Mendeleiev (Mazurs, 1974).
De otra parte, con respecto al concepto elemento, algunos libros de texto publicados pocos años después del de Mendeleiev seguían definiéndolo como sustancia, como afirmaba Davey (1927):

Toda la teoría química que se encuentra en nuestros libros de texto está basada en la suposición de que el universo material está compuesto por un número limitado de sustancias elementales llamadas "elementos" y que estos "elementos" están compuestos por unas partículas diminutas llamadas "átomos", cada uno de los cuales trata de mantener su identidad bajo todas las condiciones ordinarias de presión y temperatura, incluso cuando se encuentran en contacto íntimo como otros "átomos" de otros "elementos".

En 1937, Menschutkin publicó un artículo bajo el título de "Historical Development of the Conception of Chemical Elements". De acuerdo con Menschutkin,

...los elementos químicos (1) no se deben descomponer químicamente en sustancias más simples y (2) deben hacer parte de una sustancia dada. [...] Las sustancias simples cumplen sólo con una de las condiciones de Lavoisier: no se pueden descomponer en otras más simples pero no están contenidas en ninguna sustancia compuesta.

Más adelante, el autor continúa su explicación y refiriéndose a los "principios" de Fourcroy, añade:

Voy a mantener las dos palabras "principio" y "sustancia simple" para distinguir las dos concepciones. No hay duda de que los "principios" son los verdaderos elementos químicos ya que no se pueden descomponer 
químicamente y son los componentes últimos de los cuerpos simple y compuestos, así que satisfacen la definición de elemento químico.

Para cerrar esta discusión el autor retoma el trabajo de Mendeleiev y concluye:

Casi todos los químicos, hasta hoy, confunden los principios con las sustancias simples, designando ambos con el mismo término "elemento químico”. En el Primer Congreso de Química, en 1860, pareció como si la mayoría considerara el "principio" como elemento químico y el trabajo de Mendeleiev marcó las diferencias entre las sustancias químicas y los principios y mantuvo a estos últimos como elementos químicos. Su sistema periódico es entonces un sistema de principios, no de sustancias simples.

Basado en las resoluciones de la International Union de 1923, Menschutkin (1937, p. 61) definió elemento de la siguiente manera: "Cada grupo de átomos y sus iones, todos con el mismo número atómico, forman un 'agregado', una 'colección'. Cada uno de ellos es un elemento químico, que se puede definir así: un elemento químico es un principio cuyos átomos e iones tienen el mismo número atómico".

No obstante, hoy las diferentes definiciones de elemento siguen siendo utilizadas indiscriminadamente por profesores y textos de química (Bullejos et al., 1995; Alvadalejo et al., 1981; Roundy, 1989; Bensaude-Vincent, 1994b; Thibault et al., 1994) sin tener en cuenta que un mismo elemento puede presentarse como varios isótopos, de modo que todos los átomos de ese elemento no son necesariamente idénticos, con lo cual la definición de Dalton queda fuera de lugar. Y, por otra parte, el protio y el deuterio pueden separarse en una mezcla de agua y agua pesada por electrólisis, que es un proceso químico (Roundy, 1989), lo que hace que la definición de Boyle y la de Lavoisier también se invaliden. Podemos agregar a esto la confusión que aún subsiste entre elemento y sustancia simple o cuerpo simple, a pesar de la aclaración hecha hace más de un siglo por Mendeleiev, y en un mismo recuadro de la TP se incluyen propiedades de uno y otra, por ejemplo el número atómico del elemento y la temperatura de fusión de la sustancia simple. Al respecto, Thibault et al. (1994, p. 57), manifiestan que la IUPAC, en su sección de nomenclatura de química inorgánica en 1990, buscando aclarar esta situación, planteó:

Este capítulo concierne con uno de los formalismos básicos de la química, la representación de los elementos mediante símbolos. Generalmente, no se hace distinción entre un elemento y una sustancia elemental en los países de habla inglesa. Sin embargo, algunos consideran al primero como una abstracción mientras que la segunda es, indudablemente, una forma de materia. A menudo no está claramente indicado si un símbolo representa un átomo o un elemento. Fue considerablemente difícil obtener definiciones que satisficiesen todos los requisitos. Las definiciones que se presentan aquí pretenden ser útiles y ampliamente aplicables, aún si algunas veces pueden ser criticadas desde un punto filosófico.

Las definiciones que proponen son: un elemento (o sustancia elemental) es materia, cuyos átomos son todos iguales en cuanto a que poseen la misma carga positiva en su núcleo; un átomo 
es la cantidad unitaria más pequeña de un elemento capaz de existir sola o en combinación química con otros átomos del mismo o de otro elemento.

Por su parte, Bullejos et al. (1995) manifiestan que:

La sustancia simple es un sistema que posee un conjunto de propiedades características, tales como punto de fusión, ebullición, densidad, etc., mientras que el elemento es una clase de átomos de los que suponemos, según la teoría atómica, que están compuestas las sustancias. Los elementos no poseen propiedades características como las sustancias. [...] En los cambios químicos se conservan los elementos, esto es, los átomos, pero no se conservan las sustancias simples.

Durante más de un siglo, la TP, desde su nacimiento, se ha constituido en el punto de intersección entre la investigación y la enseñanza de la química. En palabras de Bensaude-Vincent (1995, p. 507): "Una urgencia pedagógica constituye, pues, el origen del descubrimiento (de la ley periódica)".

Por un lado, las investigaciones científicas han llevado al descubrimiento y la obtención de más de cien elementos proveyendo además toda la sustentación teórica de su organización, y por otro, su enseñanza ha motivado nuevas propuestas para la transposición de tal conocimiento (Albaladejo et al., 1982; Goth, 1986; Brincones y García, 1987; Garrigós et al., 1987; Goh y Chia, 1989; Bensaude-Vincent, 1994; Cherif et al., 1997; Ben-Zvi y Genut, 1998; Dreyfyss, 2000; Yin y Ochs, 2001), generando un enriquecimiento continuo tanto en su contenido como en su utilidad.
Los nuevos descubrimientos sobre la estructura del átomo fueron modificando a su vez el sentido de la TP aunque, en esencia, su formato se conserve muy similar al original. Lo que comenzó como una ley general de comportamiento químico se fue convirtiendo en un sistema ordenador basado en "capas de electrones" y "electrones de valencia" (Izquierdo, 2002). Sin embargo, los nuevos avances en la dilucidación de la estructura del átomo y la actualización de la TP a partir de los nuevos descubrimientos iban apareciendo en la literatura científica, pero tardaban mucho en llegar a los libros de texto y a los salones de clase (Higgins, 1926; Foster, 1939; Campell, 1946).

\section{Metodología}

Los 109 artículos encontrados bajo los indicadores TP y elemento químico fueron leídos en orden cronológico, resumidos y clasificados de acuerdo a su relación particular con el concepto elemento o con la TP. Una vez se tuvo una visión global, se analizaron los aspectos más destacados en los distintos momentos. No obstante, los resultados que se presentan a continuación constituyen un resumen de la revisión efectuada pero no incluyen una reflexión histórica social del desarrollo de tales conceptos.

\section{Resultados}

Desde el primer artículo (Sears, 1924), aparecen los dos aspectos relevantes que estarán presentes en esta búsqueda bibliográfica: el principio subyacente responsable de la periodicidad observada por Mendeleiev en el comportamiento de las propiedades de las sustancias y las propuestas para modificar o sustituir el formato original de la TP. 
El primer aspecto, que en el artículo de Sears está sólo sugerido, en el de Courtines (1925) ya es explícito: "Es el número de electrones externos al núcleo atómico, igual a la carga del núcleo, lo que determina el arreglo de los elementos en un orden definido". A partir de entonces, esta justificación de la periodicidad se repite una y otra vez: Monroe y Turner (1926), Conant (1928), Van Rysselberghe (1935), Menschutkin (1937), Ebel (1938), Luder (1939), Foster (1939), Hazlehurst (1941), Luder (1943), Wiswesser (1945), Szabo y Lakatos (1957) y Strong (1959).

No obstante, se percibe también la preocupación de algunos autores (French, 1937; Ludwig, 1992) porque las tablas periódicas propuestas realzaban más una ordenación numérica que las propiedades químicas. De todas maneras, son más los artículos que sustentan la periodicidad en la estructura atómica que aquellos que aducen razones de comportamiento químico para su justificación, como se lee en Bouma (1989) quien, al proponer su formato, manifiesta que las razones de la periodicidad son: la configuración electrónica, el principio de Pauli y otros conceptos relacionados. Así mismo señala que los desarrollos en la ciencia se demoran 25 años en ser reconocidos en los libros de texto (la tabla de Mendeleiev fue presentada por primera vez en un libro de texto en los Países Bajos, 30 años después de su publicación y más de 20 años después de que la IUPAC acordara una nomenclatura [Loening, 1984] se siguen encontrando en los libros las denominaciones A y B).

Los últimos artículos que encontramos relacionando la TP con la con- figuración electrónica, Strong (1986), Ludwig (1992) y Mabrouk (2003) están dirigidos a retomar la TP como base para el aprendizaje de las configuraciones electrónicas y ya no para justificarla a partir de ellas.

El segundo aspecto que se encuentra de manera insistente en los 78 volúmenes revisados, es la proposición de nuevos formatos para organizar los elementos químicos (Sanderson, 1964; Fernelius y Powell, 1982; Fernelius, 1986; Guenther, 1987). En 1974, Edward G. Mazurs hizo una recopilación y clasificación de los muchos propuestos hasta la fecha, pero las propuestas de formatos no se han agotado. Prueba de lo anterior son los formatos presentados por Loening (1984), Garrigós et al. (1987), Sharma (1988), Campell (1989), Bouma (1989), Laing (1989), Von Marttens Osorio y Goldsmidt (1989), Levine (1990), Carrado (1993), Treptow (1994), He y Li (1997), Dutch (1999), Balaban (1999), Marshall (2000). Esta proliferación de propuestas de formatos para la TP ha sido motivo de más de un comentario en esta revista (nota al pie del editor en Longuet-Higgins [1957, p. 30]). Por su parte, Strong (1959) anotaba: "Uno [lector] interno se da cuenta que cada autor toma un principio diferente que le parece importante para su propuesta. La inconformidad con la forma corta o larga suelen ser un motivo para que los químicos entren en el juego". Décadas después, Fernelius y Powell (1982) dedicaban su artículo a la crítica de los tres formatos más comunes: el corto, el largo y el piramidal. En él presentaban un cuadro con la revisión de 829 artículos del Chemical Abstract publicados entre 1972 y 1981, anotando que "se han he- 
cho muchas propuestas de modificación y cada autor busca resaltar un determinado aspecto". Efectivamente, como afirman Fernelius y Powell (1982), en esta revisión encontramos formatos de $\mathrm{TP}$ basados en la configuración electrónica y los números cuánticos, en isótopos, en las propiedades de las sustancias simples. Hay también algunas propuestas que sólo se refieren al tipo de letras o de colores que se debe utilizar para hacerlos más llamativos o más útiles como medio didáctico' (Sears, 1924; Graves, 1929; Hazlehurst y Fornoff, 1943, Bouma,1989) y otras que están más encaminadas a explicar la periodicidad en sí. También encontramos propuestas sobre programas académicos de química o para la enseñanza de la TP, algunas investigaciones relativas al tratamiento de la TP en los libros de texto, otros relacionados con el uso de modelos, tanto materiales como teóricos y didácticos, para explicar el átomo y la TP.

Así, los 109 analizados se clasificaron bajo once ítems, como se detalla a continuación. Algunos artículos tratan simultáneamente y en profundidad diferentes aspectos y por eso pueden aparecer más de una vez.

\section{Formato}

En estos artículos se resaltan las características del formato. Lo importante es la forma o el color. Un ejemplo de este grupo lo constituye el artículo de Graves (1929) en el que describe: "En las casillas, los sólidos están representados por letras negras, los líquidos en azul,

1 Medio didáctico en el sentido de Marqués Graells (2000): "...cualquier material elaborado con la intención de facilitar los procesos de enseñanza y aprendizaje". los gases en rojo y las tierras raras en marrón...".

Sears, G. W. (1924). A new form of periodic table as a practical means of correlating the facts of chemistry. JCE, 1(8), 173-177.

Graves, S. (1929). A periodic chart of the atoms. JCE, 6(3), 553-555.

French, S. J. (1937). Warping the periodic table. JCE, 14(12), 571-573.

Foster, L. S. (1939). Why not modernize the textbooks also? I. The periodic table. JCE, 16, 409-412.

Hazlehurst, T. H. y Fornoff, F. J. (1943). Representation of periodic properties of the elements. JCE, 20, 77-79.

Coryell, C. D. (1952). The periodic table: The 6d-5f mixed transition group. JCE, 29, 62-64.

Strong, F. C. (1959). The atomic form periodic table. JCE, 36(7), 344-345.

Sanderson, R. T. (1964). A rational periodic table. JCE, 41(4), 187-190.

Ternsrom, T. (1964). A periodic table. JCE, 41(4), 190-191.

Griff, H. K., (1964). A clockwise spiral system of the chemical elements. JCE, 41(4), 191.

Fernelius, W. C. y Powell, W. H. (1982). Confusion in the periodic table of the elements. JCE, 59(6), 504-508.

Loening, K. L. (1984). Recommended format for the periodic table of the elements. JCE, 61(2), 136.

Guenther, W. B. (1987). An upward view of the periodic table. JCE, 64(1), 9-10.

Sharma, R. C. (1988). Recommended format for the periodic table. JCE, 65, 1114.

Campbell, J. A. (1989). Let us make the table periodic. JCE, 66(9), 739-740.

Bouma, J. (1989). An application-oriented periodic table of the elements. JCE, 66(9), 741-745.

Laing, M. (1989). The periodic table - A new arrangement. JCE, 66(9), 746.

Levine, E. H. (1990). Create your own periodic table. JCE, 67(12), 1045-1046. 
Carrado, K. A. (1993). Presenting the fun side of the periodic table. JCE, 70 , 658-659.

He, F. Ch. y Li, X. Y. (1997). The periodic building of the elements: Can the periodic table be transformed into stereo? JCE, 74(7), 792-793.

Balaban, A.T. (1999). A different approach to a 3-D periodic system including stable isotopes. JCE, 76(3), 359.

\section{Átomo y configuración electrónica}

Este conjunto reúne aquellos artículos que se refieren al átomo y a los números cuánticos y, en particular, a su relación con la TP.

\section{TP basada en estructura atómica}

Estos enfatizan que la configuración electrónica es la base del ordenamiento de los elementos en la TP. Por ejemplo, Monroe y Turner (1926) afirman que: "Por tanto, los elementos pueden clasificarse en periodos de acuerdo al número de órbitas electrónicas en el átomo...”. Por su parte, Ebel (1938) manifiesta que: "Pero ya que la ley tiene que ver esencialmente con las propiedades del átomo, y ya que las propiedades del átomo están determinadas por la estructura atómica, estas pueden explicar al menos el porqué de la ley periódica...".

Courtines, M. (1925). A model of the periodic table. JCE, 2(2), 107-109.

Monroe, C. J. y Turner, W. D. (1926). A new periodic table of the elements. JCE, 3(9), 1058-1065.

Ebel, R. L. (1938). Atomic structure and the periodic table. JCE, 15, 575-577.

Luder, W. F. (1939). An improved periodic table. JCE, 16, 393-395.

Luder, W. F. (1943). Electron configuration as the basis of the periodic table. JCE, 20, 21-26.

Babor, J. A. (1944). A periodic table based on atomic number and electron configu- ration. Where to place $\mathrm{Th}, \mathrm{Pa}$ and $\mathrm{U}$ in the table. JCE, 21, 25-26.

Emerson, E. I. (1944). A chart based on atomic numbers showing the electronic structure of the element. JCE, 21, 254255.

Wagner, H. A. y Simmons, B. H. (1945). A new periodic table. JCE, 22, 128-129.

Szabo, Z. G. y Lakatos, B. (1957). A new periodic table and new periodic functions. JCE, 34(9), 429-432.

Fernelius, W. C. (1986). Some reflections on the periodic table and its use. JCE, 63(3), 263-266.

\section{De la tabla a la configuración electrónica}

Estos artículos retoman la TP para reforzar los conocimientos de la química y la estructura atómica. En este sentido el artículo de Ludwig (1992) señala que: "Usar la TP exclusivamente para racionalizar la distribución electrónica tiene la ventaja de enfatizar en los aspectos experimentales de la ciencia y evitar dar la impresión de que la química es nada más que un conjunto de reglas misteriosas".

Strong, J. A. (1986). The periodic table and electron configurations. JCE, 63(10), 834-836.

Ludwig, O. G. (1992). The best Aufbau mnemonic: the periodic table. JCE, 69, 430.

Mabrouk, S. T. (2003). The periodic table as a mnemonic device for writing electronic configurations. JCE, 80(8), 894-898.

\section{Otras propuestas de tabla periódica}

Esta tabla está basada en átomos gaseosos libres y en los términos espectroscópicos para describir los átomos en su estado fundamental.

Treptow, R. S. (1994). The periodic table of the atoms. Arranging the elements by a different set of rules. JCE, 71(12), 1007-1012. 


\section{Desarrollo de la teoría atómica}

Estos artículos incluyen algo de historia sobre la teoría atómica o sobre los desarrollos en los descubrimientos de las partículas subatómicas y algunas propuestas para enseñarla.

Huggins, M. L. (1926). The structure of matter: a brief review of present day conceptions. I. The atom. JCE, 3(10), 1110-1116.

Conant, J. B. (1928). Atoms, molecules, and ions. JCE, 5(1), 25-35.

Campbell, J. A. (1946). Atomic size and the periodic table. JCE, 23, 525-529.

Standen, A. (1947). The continued fairy story of atomic weights. JCE, 24, 453.

Leary, J. J. y Kippeny, T. C. (1999). A framework for presenting the modern atom. JCE, 76(9), 1217-1218.

Hoffman, D. C. y Lee, D. M. (1999). Chemistry of the heaviest elements - one atom at a time. JCE, 76(3), 331-347.

\section{Visión de átomo}

El artículo resume una investigación realizada con 300 estudiantes de 15 años para conocer sus ideas sobre el modelo de átomo. Para ello se les preguntaba cuáles propiedades de seis propuestas creían que podría tener un átomo de cobre sólido o gaseoso. Las respuestas son el resultado de cinco modelos conceptuales.

Ben-Zvi, R.; Eylon, B. S. y Silberstein, J. (1986). Is an atom of copper malleable? JCE, 63(1), 64-66.

\section{El núcleo y la tabla periódica}

Estos artículos están directamente relacionados con propiedades del núcleo, como su estabilidad, o con espectros de resonancia magnética nuclear.

Blanck, H. F. (1989). Predicting nuclear stability using periodic table. JCE, 66(9), 757-758.
Huizenga, J. R. (1993). Size of the periodic table. Answering a philosophical question about possibilities and limitations. JCE, 70, 730-731.

Gerotthanassis, I. P. y Kalodimos, C. G. (1996). NMR Shielding and the periodic table. JCE, 73(8), 801-804.

\section{Números cuánticos}

En estos artículos se relacionan los números cuánticos con la TP o se narran aspectos de interés sobre su historia.

\section{Historia de los números cuánticos}

Este segundo artículo de Higgins plantea que gran parte del conocimiento que en ese momento se tiene sobre el átomo se ha obtenido por los estudios de las radiaciones de la luz.

Huggins, M. L. (1926). The structure of matter: a brief review of present day conceptions. II. The atom and radiation. JCE, 3(11), 1254-1258.

\section{Números cuánticos y TP}

Esta serie de artículos relaciona la TP con los números cuánticos de una forma más específica que los del apartado tabla periódica basada en estructura atómica. Por ejemplo, Van Rysselberghe (1935) afirma que "...las propiedades químicas de los elementos dependen de la naturaleza (por ejemplo, los números cuánticos) y en el número de electrones en las distintas capas de valencia. Las propiedades químicas dependen más del número cuántico azimutal, l, del electrón añadido que del número cuántico principal, n...".

Van Rysselbeerghe, P. (1935). A new periodic table. JCE, 12, 474-475.

Hazlehurst, T. H. (1941). Quantum numbers and the periodic table. JCE, 18 , 580-581. 
Wiswesser, W. J. (1945). The periodic system and the atomic structure. III. Wave mechanical interpretations. JCE, 22, 418-426.

Simmons, L. M. (1947). A modification of the periodic table. JCE, 24, 588-591.

Longuet-Higgins, H. C. (1957). A periodic table. The "Aufbauprinzip" as a basis for classification of the elements. JCE, 34(1), 30-31.

Blinder, S. M. (1981). Quantum Chemistry via the Periodic Law. JCE, 58(10), 761763.

\section{Isótopos}

Aquí se explican las anomalías de la TP en función de las diferencias en los números de masa de los isótopos. Otros proponen modelos de tabla para mostrar la abundancia de los distintos isótopos. King, J. F. y Fall, P. H. (1940). Radioactivity and the periodic table. An introduction to the study of atomic structure and isotopes in elementary chemistry. JCE, 17, 481-482.

Firsching, F. H. (1981). Anomalies in the periodic table. JCE, 58(6), 478-479.

Balaban, A. T. (1999). A different approach to a 3-D periodic system including stable isotopes. JCE, 76(3), 359.

\section{Tabla periódica y propiedades de las sustancias}

Este conjunto de artículos se refiere a propiedades de las sustancias asociadas a la TP. Ternsrom (1964), por ejemplo, propone una TP que separa los elementos de acuerdo con su estado de agregación.

Szabo, Z. G. y Lakatos, B. (1957). A new periodic table and new periodic functions. JCE, 34(9), 429-432.

Ternsrom, T. (1964). A periodic table. JCE, 41(4), 190-191.

Singman, C. N. (1984). Atomic volume and allotropy of the elements. JCE, 61(2), 137-142.
Dutch, S. I. (1999). Periodic tables of elemental abundance. JCE, 76(3), 356-358. Marshall, J. L. (2000). A Living periodic table. JCE, 77(8), 979-983.6.

\section{Ley o periodicidad}

Estos artículos presentan propuestas para enseñar la periodicidad, o se refieren a la variación de algunas propiedades periódicas. En particular, Moore (2003) enfatiza la importancia de la ley periódica afirmando que la importancia del trabajo de Mendeleiev no fue descubrir la TP sino hacer una clasificación periódica. Por tanto, continúa diciendo, no se debe hablar de la TP, sino de una TP pues hay muchas. Cita a Van Sprosen y a Mazurs, y sugiere que en la enseñanza se utilice más de una.

\section{Periodicidad}

Goh, N. K. y Chia, L. S. (1989). Using the learning cycle to introduce periodicity. JCE, 66(9), 747-749.

Rayner-Canham, G. (2000). Periodic patterns. JCE, 77(8), 1053-1056.

Moore, J. W. (2003). Turning the (periodic) tables. JCE, 80(8), 847.

\section{Propiedades periódicas}

Mason, J. (1988). Periodic contractions among the elements. Or, on being the right size. JCE, 65(1), 17-20.

Cann, P. (2000). Ionization energies, parallel spins, and the stability of half-field shells. JCE, 77(8), 1056-1061.

\section{Modelos}

Bajo el encabezamiento de modelos se han agrupado tanto los artículos referentes a modelos materiales, como los teóricos y didácticos.

Modelos materiales: un ejemplo representativo de este grupo es el artículo de Re- 
cords (1982) en el que el autor presenta propuestas para hablar en secundaria de los cinco modelos atómicos más comunes: a) las esferas uniformes de Dalton; b) el budín con pasas de Thomson; c) el modelo nuclear de Rutherford; d) el de niveles de energía de Bohr, y e) el modelo orbital de la mecánica cuántica.

Campbell, J. A. (1946). Atomic size and the periodic table. JCE, 23, 525-529.

Sanderson, R. T. (1960) Atomic models in teaching chemistry. JCE, 37(6), 307-310.

Records, R. M. (1982). Developing Models: What is the atom really like? JCE, 59(4), 307-309.

Smith, R. (1989). The historic atom: from D to Q. JCE, 66(8), 637-638.

He, F. Ch. y Li, X. Y. (1997). The periodic building of the elements: Can the periodic table be transformed into stereo? JCE, 74(7), 792-793.

Modelos teóricos: en este artículo el autor hace una descripción de los modelos atómicos a partir de Bohr y Rutherford. Cita a personajes de la física y la química del siglo XX y transcribe apartes de sus textos para realzar los modelos o las aportaciones a los modelos atómicos.

Wiswesser, W. J. (1948). Atomic structure models, diagrams, classes, and codes. Part I. Which atomic Model? Challenge to teachers. JCE, 25, 420-425.

Modelos didácticos: este conjunto de artículos consiste en propuestas para llevar a cabo procesos de modelización en la clase. Como ejemplo podemos citar el artículo de Schrader (1985) en el que el autor describe algunas de sus prácticas tanto en el laboratorio como en la clase para que los estudiantes "modelicen" algunos conceptos. En alguno de ellos siguen los mismos pasos que Mendeleiev y llegan a conclusiones similares. La conclusión es que cuando los estudiantes entienden lo que es un modelo, pueden estar más abiertos a las nuevas teorías y hechos de la ciencia.

Fowler, L. S. (1981). Building a periodic table. JCE, 58(8), 634-635.

Schrader, C. L. (1985). Everyone wants to be a model teacher. Part III. Extensions to atomic structures and bonding. JCE, 62(1), 71-72.

Strong, J. A. (1986). The periodic table and electron configurations. JCE, 63(10), 834-836.

Ciparick, J. D. (1988). Introduction to atomic structure: demonstrations and labs. JCE, 65, 892-893.

Bolmgren, I. (1995). Presenting the periodic system with pictures. JCE, 72(4), 337-338.

\section{Libros}

Estos tres artículos hacen referencia a investigaciones hechas en libros de texto, aunque sea de modo colateral o complementario al tema central de su artículo. Por ejemplo, Fernelius y Powell (1982) presentan un cuadro sobre las tablas periódicas usadas por 195 libros de texto de química entre 1931 y 1981. La más usada es la tabla larga.

Huggins, M. L. (1926). The structure of matter: a brief review of present day conceptions. I. The atom. JCE, 3(10), 1110-1116.

Hazlehurst T. H. (1941). Quantum numbers and the periodic table. JCE, 18, 580-581.

Fernelius, W. C. y Powell, W. H. (1982). Confusion in the periodic table of the elements. JCE, 59(6), 504-508.

\section{Programas y propuestas de química}

Bajo este encabezado hemos reunido las propuestas para los cursos de química, para la enseñanza de algunos temas en particular y para la carrera de química. 
Programas para un curso de química general: esta primera serie de artículos busca responder, cada uno a su manera, la pregunta propuesta por Tamres y Bailar Jr. (1952): “¿Se puede diseñar un solo curso para suplir todas las necesidades de todos los estudiantes?".

Urey, H. C. (1929). The teaching of atomic structure to physical chemists: Roundtable discussion. JCE, 6(2), 284-285.

Wiswesser, W. J. (1945). The periodic system and the atomic structure. I. An elementary physical approach. JCE, 22, 314-321.

Sisler, H. H. (1948). Why should theoretical principles be used in teaching elementary chemistry? JCE, 25, 562- 565.

Tamres, M. y Bailar, J.C., Jr. (1952). The course in general chemistry. JCE, 29, 217-219.

Whitman, M. (1984). Updating the atomic theory in general Chemistry. JCE, 61(11), 952-956.

Woodgate, S. D. (1995). First-year chemistry in the context of the periodic table. JCE, 72(7), 618-622.

Glickstein, N. (1999). Before there was chemistry: The origin of the elements as an introduction to chemistry. JCE, 76(3), 353-355.

Waldron, K. A.; Fehringer, E. M.; Streeb, A. E.; Trosky, J. E. y Pearson, J. J. (2001). Screening percentages based on Slater effective nuclear charge as a versatile tool for teaching periodic trends. JCE, 78(5), 635-639.

Yin, M. y Ochs, R. S. (2001). The mole, the periodic table, and quantum numbers: an introductory trio. JCE, 78(10), 1345-1347.

Programas para la carrera química (currículo): En este artículo se comenta sobre la celebración de los 75 años de American Chemical Society y se discute sobre los cambios que ha habido tanto a nivel de la química como de la industria química.

Elving, P. J. (1952). The curriculum in chemistry. JCE, 29, 216-217.

Propuestas para la enseñanza: En este apartado se encuentran artículos con propuestas para la enseñanza de un tema específico o se narran experiencias particulares.

De la tabla periódica: Estos tres artículos presentan: una propuesta para una base de datos sobre la TP, una TP hecha con sellos de correos diseñados por los estudiantes y la experiencia de un profesor que prestó su coche para que sus estudiantes pintaran la TP en él y la pasearan por toda la ciudad.

Goth, G. W. (1986). The periodic table as a data base. JCE, 63(10), 836-837.

Garrigós Ll., Ferrando, F. y Miralles, R. (1987). A simple postage stamp periodic table. JCE, 64(8), 682-685.

Dreyfuss, D. (2000). A rolling periodic table. JCE, 77(4), 434.

De reacciones nucleares: Los autores comentan un juego de mesa para que los estudiantes aprendan sobre las reacciones nucleares, tanto síntesis como decaimiento.

Olbris, D. J. y Herzfeld J. (1999). Nucleogenesis! A game with natural rules for teaching nuclear synthesis and decay. JCE, 76(3), 349-352.

\section{Historia o filosofía}

El siguiente grupo de artículos hace referencia a la historia del átomo o de la TP y a la importancia de los aspectos históricos y filosóficos en la química y su enseñanza. 
Átomo (historia): Varios de ellos hacen un recuento histórico del desarrollo de la teoría atómica y algunos además destacan la estrecha relación entre la ontogenia y la filogenia. En este sentido, Horne (1958) afirma que indudablemente el desarrollo del conocimiento científico en niños y en las culturas siguen una trayectoria natural similar, sugiriendo una eficacia especial en la presentación histórica de las ciencias naturales.

Foster, W. (1926). From atom to life. JCE, 3(12), 1391-1401.

Davey, W. P. (1927). The reality of atom. JCE, 4(3), 327-332.

Wiswesser, W. J. (1945). The periodic system and the atomic structure. II. Detailed introduction to the wave mechanical approach. JCE, 22, 370-379.

Scott, J. H. (1959).The nineteenth century atom: Undivided or Indivisible? JCE, 36, 64-67.

Horne, R. A. (1958). Atomistic notions in young children and young cultures. JCE, 35, 560-561.

Gorman, M. (1960). Philosophical antecedents of the modern atom. JCE, 37(2), 100-104.

Kolb, D. (1977). But if atoms are so tiny... J. of Chemical Education. JCE, 54(9), 543-547.

Ierzecki, R. (1981). Dalton's atoms or Dalton's molecules? JCE, 58, 1006.

Leary, J. J. y Kippeny, T. C. (1999). A framework for presenting the modern atom. JCE, 76(9), 1217-1218.

Hoffman, D. C. y Lee, D. M.(1999). Chemistry of the heaviest elements - one atom at a time. JCE, 76(3), 331-347.

Tabla periódica (historia): Estos artículos narran distintos aspectos de la historia que llevó a la TP de Mendeleiev. Algunos destacan otras propuestas como las de Döbereiner o Newlands, otros cuentan sobre la equivocada inclusión del éter en la tabla, por parte de Mendeleiev y otros hacen una evaluación resumida de los distintos formatos de TP propuestos a lo largo del tiempo.

Montgomery, J. P. (1931). Döbereiner's triads and atomic numbers. JCE, 8(1), 168.

Standen, A. (1947). The fairy story of atomic weights. JCE, 24, 143-145.

Taylor, W. H. (1949). J.A.R. Newlands: a pioneer in atomic numbers. JCE, 26, 491-496.

Van Spronson, J. W. (1959). The prehistory of the periodic system of the elements. JCE, 36(7), 565-567.

Anders, O. U. (1964). The place of isotopes in the periodic table. The 50th anniversary of the Fajans-Soddy displacement laws. JCE, 41(10), 522-525.

Kargon, R. (1965). Mendeleiev's chemical ether, electrons, and the atomic theory. JCE, 42(7), 388-389.

Fernelius, W. C. (1986). Some reflections on the periodic table and its use. JCE, 633), 263-266.

Elemento (historia): Menschutkin hace un recuento de la historia del elemento químico hasta la última definición aceptada en su momento.

Menschutkin, B. N. (1937). Historical development of the conception of chemical elements. JCE, 14, 59-61.

Filosofia: El autor hace una comparación paralela entre la cadena del ser (o la cadena de los seres vivos) y los elementos químicos.

Benfey, O. T. (1965). "The great chain of being" and the periodic table of the elements. JCE, 42(1), 39-41.

Otras historias: El autor cuenta cómo Thomas Midgley se basó en la TP para encontrar un aditivo antidetonante que mejorara la eficiencia de las máquinas. Jocosamente dice que probó más de 
33.000 compuestos, siguiendo lo que él mismo denominó un acercamiento al método edisoniano.

Nagel, M. C. (1981). Dr. Thomas Midgley: "From the periodic table to production". JCE, 58(6), 496-497.

\section{Analogías, cuentos y poemas}

En este último apartado se recogen algunas analogías, cuentos y poemas sobre los temas de interés en este trabajo.

Cuentos y poemas: En el primer artículo (Erwin, 1925), la autora hace la narración de una fiesta infantil a la que han sido invitados los elementos para celebrar la fiesta de cumpleaños del hidrógeno. Organizan un juego en el que todos han de acomodarse de acuerdo con sus pesos en siete filas de ocho asientos cada una.

Erwin, E. (1925). The periodic law. A bedtime story for wide-awake children. JCE, 2(6), 497-498.

Johnson, E. N. (1970). What's an atom? JCE, 47(7), 500.

Williams, K. R. y Myers, G. H. (1999). The Cinderella Story revisited - again. JCE, 76(1), 19.

Analogías: A continuación aparecen los artículos relacionados con las analogías, algunas de las cuales también fueron propuestas por los profesores de nuestra investigación.

Estructura atómica. Los autores presentan la analogía del salón de clase para explicar los números cuánticos.

Khang Goh, N.; Sai Chang, L. y Tan, D. (1994). Some analogies for teaching atomic structure at the high school level. JCE, 71(9), 733-734.
Mol, tamaño y masa atómica promedio. En general son analogías propuestas para facilitar el aprendizaje de algunos conceptos específicos.

Alexander, M. D.; Ewing, G. J. y Abbott, F. T. (1984). Analogies that indicate the size of atoms and molecules and the magnitude of Avogadro's number. JCE, 61(7), 591.

Arce de Sanabria, J. (1993). Relative atomic mass and the mole: A concrete analogy to help students understand these abstract concepts. JCE, 70(3), 233-234.

Last, A. M. (1993). Using monetary analogies to teach average atomic mass. JCE, 70(3), 234-236.

Los cuadros 1,2 y 3 condensan la información recogida en este apartado.

\section{Conclusiones}

- El concepto elemento sigue siendo utilizado en la literatura científica para referirse explícita o implícitamente a diferentes conceptos relacionados con él. Dentro de los artículos revisados, sólo Menschutkin (1937) define explícitamente elemento, aunque otros discutan tangencialmente las definiciones históricas. No obstante, a pesar de no estar explícita, esta polisemia del concepto elemento sí se trasluce en el Journal of Chemical Education (JCE) en la variedad de tablas encontradas basadas en aspectos tan diferentes como el estado de agregación (Ternsrom, 1964; Marshall, 2000), las estabilidades nucleares o la desintegración de elementos radioactivos (King y Fall, 1940) o la química de "un solo átomo" (Hoffman y Lee, 1999). Los libros de texto reflejan esta misma pluralidad (Roundy, 1989; Thibault et al., 1994; Bullejos et al., 1995). 


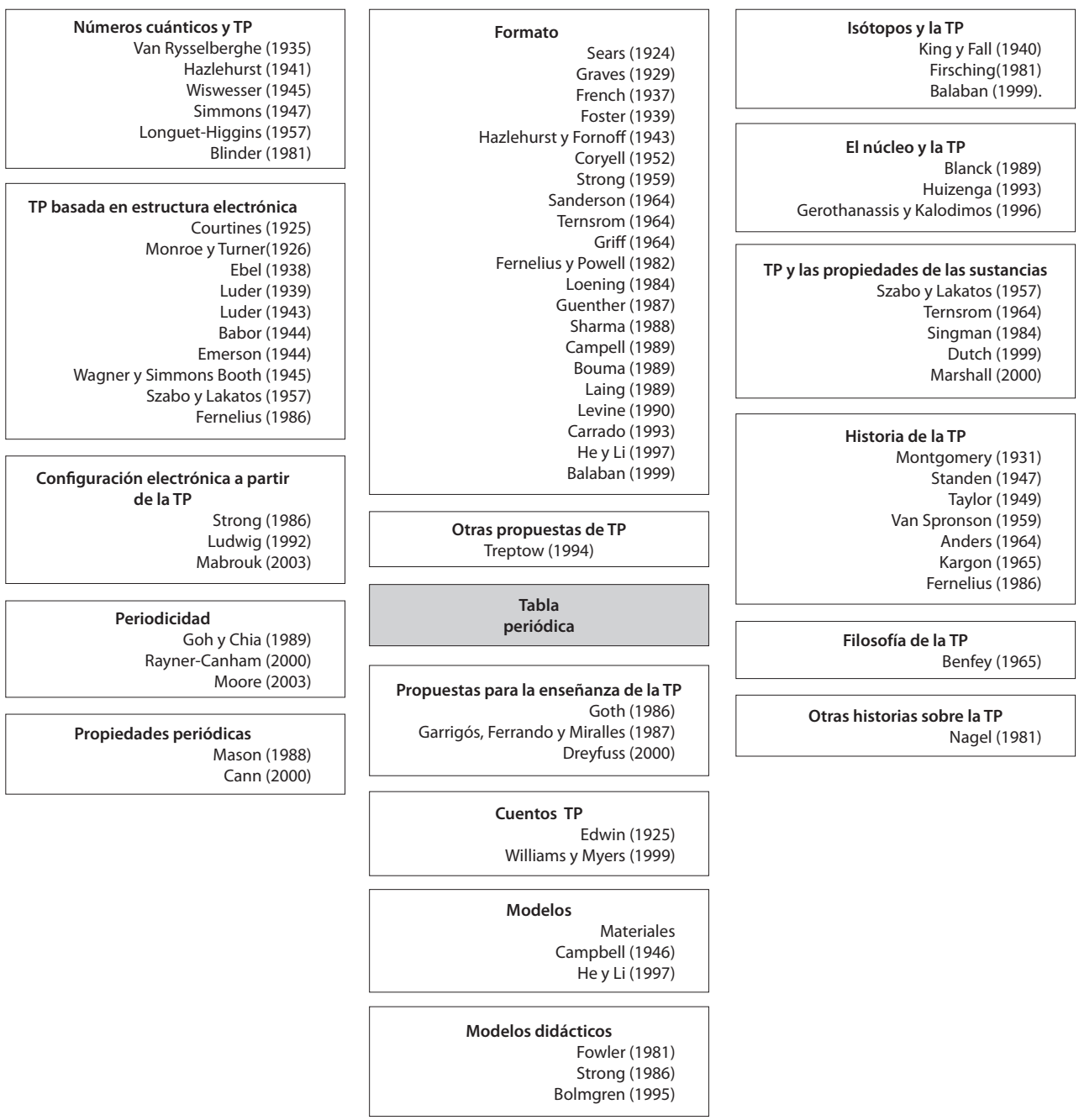

Cuadro 1. Artículos sobre la tabla periódica

- La TP se relaciona con los desarrollos científicos, en particular con la estructura atómica desde dos retóricas diferentes. En los primeros años de la revista, los autores, con muy pocas excepciones, sustentan la periodicidad en la estructura atómica (Courtines, 1925; Monroe y Turner, 1926; Ebel, 1938; Luder, 1939; Foster, 1939; Luder, 1943; Wiswesser,
1945; Szabo y Lakatos, 1957; Strong, 1959). En cambio, los artículos más recientes apoyan el aprendizaje de la estructura en la TP (French, 1937; Ludwig, 1992).

- Concernientes al contenido de los cursos generales encontramos los artículos de Urey (1929), Wiswesser (1945), Sisler (1948), Tamres y Bailar Jr. (1952), Whitman (1984), Wood- 


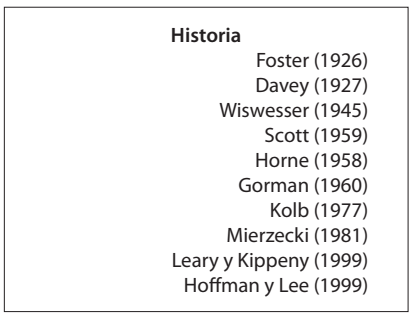

Historia de los números cuánticos Huggins (1926)

Cuadro 2. Artículos sobre el átomo.
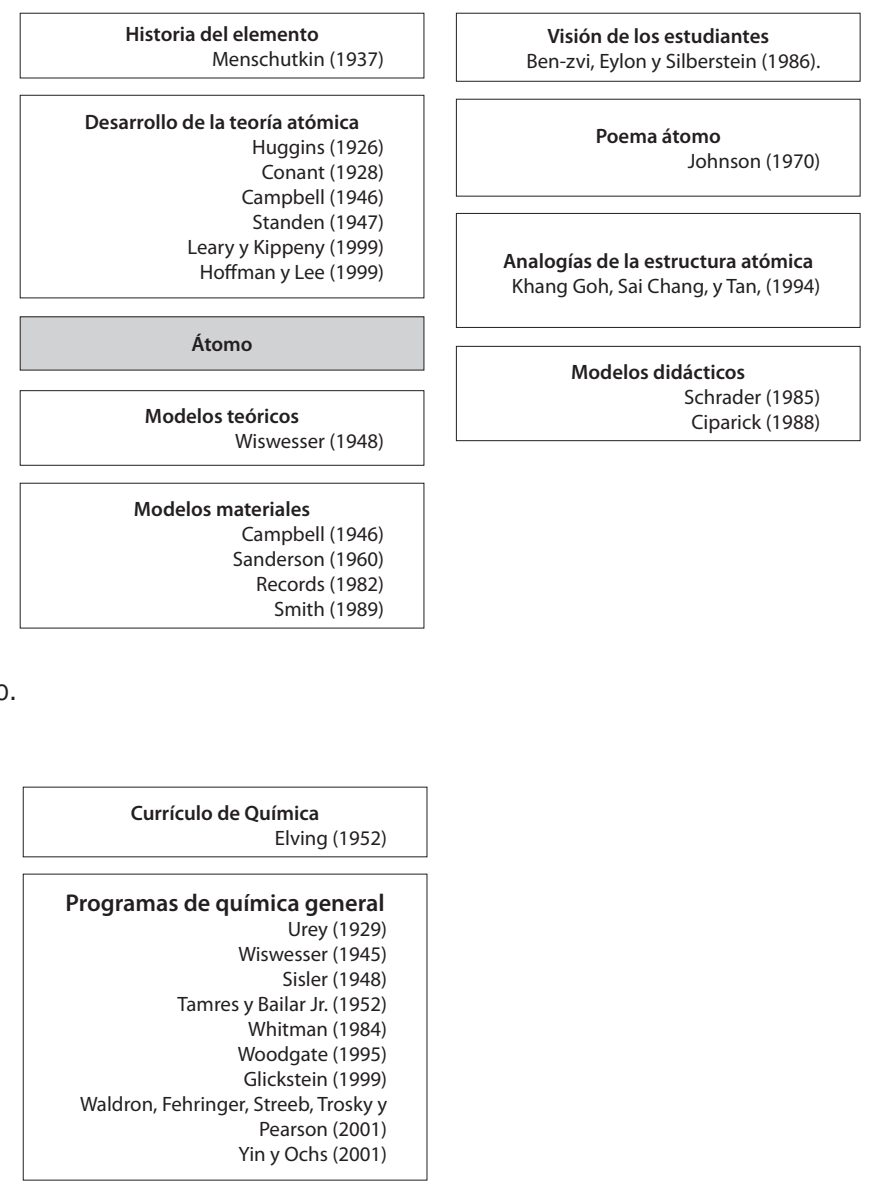

Cuadro 3. Artículos sobre programas y propuestas de la química.

gate (1995), Waldron et al. (2001). Todos son partidarios de enseñar los fundamentos teóricos de manera rigurosa desde los primeros cursos universitarios. En cuanto al modelo atómico encontramos tres tipos de artículos diferentes: unos que describen modelos materiales como los de Records (1982) y Smith (1989), para facilitar la visualización de los distintos modelos atómicos. El de Ciparick (1988) hace referencia a algunas experiencias prácticas que permiten probar la existencia de los electrones para que los estudiantes infieran la estructura atómica y asocien sus conceptos básicos a la realidad. El tercer tipo de artículos relacionado con los modelos atómicos se centran más en el aspecto teórico de la fundamentación conceptual (Urey, 1929; Wiswesser, 1948; Whitman, 1984).

En cuanto al uso de recursos para la enseñanza de la TP hay dos analogías propuestas por Goh et al. (1989, 1994). La primera se basa en el uso de un alma- 
naque para explicar la periodicidad. La segunda explica la probabilidad de encontrar un electrón en una determinada región del espacio comparándola con la ubicación de un estudiante en un salón de clase. También encontramos el uso de hojas de trabajo o de "juegos de razonamiento” propuestos por Fowler (1981), Strong (1986) y Mabrouk (2003).

\section{Bibliografía}

Alvaladejo, E. et al. (1981). ¿Cómo diferenciar entre elementos y compuestos? Cuadernos de pedagogía, 7(78), 70-72.

Bensaude-Vincent, B. (1994b). Le langage chimique a la recherche de l'élément chimique. La actualité chimique, julioagosto, 51-55.

Bensaude-Vincent, B. y Stengers, I. (1997). Historia de la Química. Madrid: Adison-Wesley Iberoamericana-Universidad Autónoma de Madrid.

Bullejos, J.; De Manuel, E. y Furió, C. (1995). ¿Sustancias simples y/o elementos? Usos del término elemento químico en los libros de texto. Didáctica de las ciencias experimentales y sociales, 9, 27-42.

Izquierdo, M. (2002). Cambio de paradigma en la Química del siglo XXI. La sorprendente evolución de la tabla periódica. Conferencia. Reunión Anual de la Sociedad Española de
Por otra parte, lo que surge de este trabajo es que para poder comprender química y significativamente lo que es un elemento químico se requiere de la interrelación del pensar, el decir y el hacer, y la TP es el recurso didáctico ${ }^{2}$ más útil para organizar, explicar y comprender todo el conocimiento que ella encierra acerca de esos elementos químicos. $\boldsymbol{\Delta}$

Historia de la Ciencia y de la Técnica (SEHCYT), Logroño.

Kolodkine, P. (1963). Dimitri Mendeleiev y la tabla periódica de los elementos. Madrid: Ediciones CID.

Mazurs, E. G. (1974). Graphic representations of the periodic system during one hundred years. 2. ${ }^{\text {a }}$ ed. EE. UU.: The University of Alabama Press.

Marquès Graells. (2000). Los medios didácticos y los recursos educativos. Consultado en: http://dewey.uab.es/ pmarques/medios.htm

Scerri, E. (1998). Evolución del sistema periódico. Investigación y Ciencia, 266, 54-59.

Thibault, J.; Figuière, P.; Legendre, J.J.; Pacault, A. y Tiberghien, A. (1994). L'élément chimique dans les écrits et dans les esprits contemporains. La actualité chimique, julio-agosto, 56-57.

2 Recurso didáctico en el sentido de Marquès Graells (2000): "...cualquier material que, en un contexto educativo determinado, sea utilizado con una finalidad didáctica o para facilitar el desarrollo de las actividades formativas". 